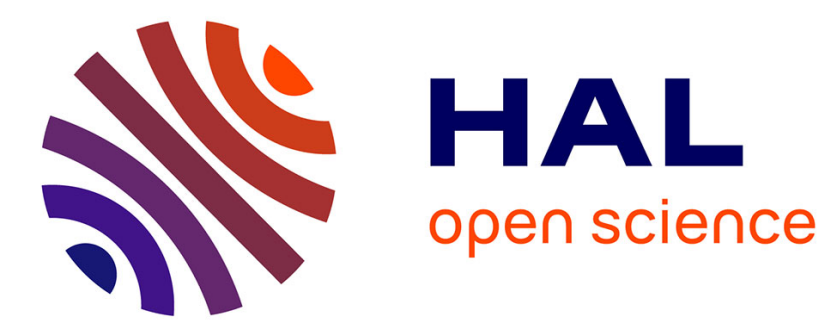

\title{
Les audiences du 35 quater. Une procédure de jugement à la frontière
}

Chowra Makaremi

\section{To cite this version:}

Chowra Makaremi. Les audiences du 35 quater. Une procédure de jugement à la frontière. Les nouvelles frontières de la société française, La Découverte, pp.437-458, 2010. hal-03038396

\section{HAL Id: hal-03038396 https://hal.science/hal-03038396}

Submitted on 29 Jan 2021

HAL is a multi-disciplinary open access archive for the deposit and dissemination of scientific research documents, whether they are published or not. The documents may come from teaching and research institutions in France or abroad, or from public or private research centers.
L'archive ouverte pluridisciplinaire HAL, est destinée au dépôt et à la diffusion de documents scientifiques de niveau recherche, publiés ou non, émanant des établissements d'enseignement et de recherche français ou étrangers, des laboratoires publics ou privés. 
Les audiences du 35 quater. Une procédure de jugement à la frontière

\author{
Chowra Makaremi
}

D'après l'article 221-1 du Code de l'entrée et du séjour des étrangers et du droit d'asile (Ceseda), trois types de voyageurs peuvent être refoulés aux frontières françaises : les personnes qui n'ont pas les documents requis pour entrer régulièrement ou qui sont soupçonnés de migration irrégulière au-delà du terme de leur visa, les personnes qui transitent par la France mais qui n'ont pas de " visa de transit » ou sont refusés par leur pays de destination, et les demandeurs d'asile. Dans ce dernier cas toutefois, la demande d'asile doit d'abord être examinée par le ministère de l'Intérieur : la Convention de Genève de 1951 sur les réfugiés, à laquelle la France est partie, dispense en effet les demandeurs d'asile de présenter les documents exigibles des étrangers pour entrer dans un pays auquel ils demandent protection. Lorsque les voyageurs arrivent en train, en bateau ou en avion, une difficulté particulière tient à ce qu'ils franchissent la frontière non pas aux limites mais à l'intérieur du territoire : la police qui leur refuse l'entrée ne peut donc pas les refouler immédiatement de l'autre côté de la frontière car, là où ils se trouvent, il n'y a pas d'autre côté. C'est pour répondre à ce paradoxe que se sont mis en place des espaces frontaliers fermés, qui relèvent d'un statut juridique spécial ${ }^{1}$ les définissant comme étant situés «à la frontière », c'està-dire soustraits aux règles de droit qui s'appliquent sur le territoire et soumis à une réglementation spécifique. Les personnes refusées y sont gardées en attendant leur refoulement ou leur admission. Ces «zones d'attente» sont le plus souvent des espaces ad hoc, affectés à cette fonction lorsque le besoin s'en fait sentir. Par exemple, lorsque l'East-Sea, un navire de marchandises battant pavillon cambodgien et transportant notamment plusieurs centaines de demandeurs d'asile kurdes irakiens, s'est échoué à Boulouris, près de Fréjus, en 2001, des barrières furent dressées autour d'une portion de plage déclarée « zone d'attente » par le préfet du Var. Dans un cas toutefois, celui de l'aéroport de Roissy, où arrivent $59 \%$ des personnes maintenues aux frontières françaises (et $79 \%$ de celles maintenues aux frontières aériennes), la frontière a évolué vers des pratiques de gestion pérennes, aux-

\footnotetext{
${ }^{1}$ Institué par la loi Quilès du 26 juillet 1992.
} 
quelles s'intéresse mon enquête.

Le dispositif d'enfermement et de traitement administratif des étrangers refusés à Roissy n'est pas un phénomène isolé : il renvoie à l'institution répandue de camps frontaliers dans les pays occidentaux. De nombreuses études empiriques menées dans ces dernières années ont documenté comment ces espaces reconfiguraient les frontières, comprises comme lieux concrets de passage et d'accès à un territoire, en zones habitées, possédant leurs propres temps et espace et leurs règles de gestion [Welch et Schuster, 2005 ; Fischer, 2005 ; Rahola, 2007 ; Courau, 2007]. Au regard de ce phénomène commun aux pays de l'Union Européenne et à leurs voisins [Bauer et Darley, 2007; Andrijasevic, 2006], que l'on retrouve notamment en Amérique du Nord [Pratt, 2005], en Australie [Mares, 2001] et en Israël [Willen, 2008], la France se distingue particulièrement par la complexité de la procédure de détention et la façon dont celle-ci est investie par le droit. Ce traitement administratif prend forme dans le va-et-vient entre la procédure de sélection et de contrôle aux frontières et les différentes instances de jugement, incluses dans cette procédure ou mobilisées par les étrangers et leurs défenseurs.

La détention administrative en zones d'attente est soumise, depuis 1992, au contrôle d'un juge qui en vérifie la légalité. Le passage devant le juge des libertés et de la détention (JLD), qui a le pouvoir de prolonger la détention ou d'admettre l'étranger sur le territoire, est ainsi devenu une étape du maintien aux frontières, chargée d'enjeux juridiques et pratiques. Ce chapitre souhaite réfléchir sur ces enjeux à travers l'exercice quotidien de la justice, son évolution dans le temps et les discours qui la construisent. Comment le contrôle juridique qui se met en place avec la légalisation des zones d'attente ressaisit-t-il la frontière, en tant que lieu particulier de gestion de non-citoyens, comme un espace sous contrôle du droit? Une observation de l'institution et de l'exercice de ce contrôle pointe les tensions, les discours, les ruses et les usages qui ont construit le quotidien d'une frontière comme procédure et comme jugement. Ces processus montrent comment la construction des frontières dans l'espace du politique relève d'une négociation constante à l'intérieur de cet espace, avec ses pratiques et ses apories. Particulièrement intéressante en ce sens est la question de la confidentialité ou au contraire de la publicité du travail des juges, autour de laquelle se nouent les enjeux de localisation des audiences au sein du dispositif frontalier, et sur laquelle ce chapitre reviendra plus longuement. 
in D. Fassin (dir.), Les nouvelles frontières de la société française, Paris, La Découverte, 2010, pp. $437-458$

\section{Le contrôle juridique : aux origines de la zone d'attente}

Jusqu'en 1992, les étrangers refusés à Roissy sont détenus dans n'importe quel lieu disponible (postes de police, guichets de contrôle de douane, salles d'attente des compagnies aériennes, hangars) pourvu qu'il se trouve en zone internationale. Cette zone appelée aussi zone sous douane désigne l'espace situé avant les postes de contrôle de la Police de l'air aux frontières (PAF). Juridiquement, le ministère de l'Intérieur français le considère comme extraterritorial : la loi française ne s'y applique pas, aucune régulation particulière n'encadre la durée, ni les conditions et les voies de recours contre la détention administrative qui s'y installe.

Alors que les politiques de contrôle migratoire se traduisent notamment par un contrôle accru des frontières à partir des années $1980^{2}$, l'augmentation du nombre d'étrangers et de demandeurs d'asiles gardés en zone internationale rend leur condition visible aux salariés de l'aéroport et des compagnies aériennes, comme le rapporte un responsable syndical alors en fonction à Roissy : "Des agents de transit étaient relativement choqués par les mesures de reconduite à la frontière de familles, de réfugiés économiques ou politiques et qu'on n'acceptait pas. [...] le spectacle auquel assistaient mes collègues était quelque chose d'assez désastreux de violence policière et de ces gens qui ont tout misé pour refaire leur vie ailleurs et qu'on oblige à repartir dans leur pays... avec les risques que ça comporte, parce qu'on s'est beaucoup interrogé : quand ces personnes revenaient dans leur pays, on n'avait pas de nouvelles, on ne savait pas ce qui se passait. Est-ce qu'elles n'ont pas été mises en prison ? [...Un jour], on est arrivé dans une salle qui était dans le terminal A et qui était en zone sous douane. La salle était grande de quelques mètres carrés et vous aviez là-dedans une trentaine de personnes dans une chaleur étouffante, des femmes, des enfants. Les hommes urinaient dans des bouteilles en plastique, certains étaient malades. Quand on est arrivé, il y en avait un qui avait l'oreille en sang, il avait été frappé par un policier, il n'entendait plus rien. C'était arrivé quelques temps avant et il n'avait pas été soigné, il y avait encore quelques traces de sang. Et là c'était... la misère du monde, c'était insupportable; et les plateaux [de repas servis aux personnes enfermées] qui s'entassaient partout. Beaucoup étaient malade, avaient des diarrhées. Les policiers ne les laissaient pas aller aux toilettes, en disant: "Ils exagèrent de vouloir tout le temps aller aux toilettes". Il n'y avait pas de couches pour les enfants.

\footnotetext{
2 En 1986 et 1987, des circulaires du ministère de l'Intérieur mettent en place un rouage essentiel du contrôle aux frontières en attribuant à la police un pouvoir d'appréciation discrétionnaire sur les pièces d'identité, les justificatifs de conditions du séjour et les motifs de la venue des étrangers, qui sont désormais contrôlés.
} 
in D. Fassin (dir.), Les nouvelles frontières de la société française, Paris, La Découverte, 2010, pp. 437-458

Franchement, un traitement inhumain. [...] La salle était à quelques mètres de là où circulent les passagers. Ils avaient fermé sous l'escalier une zone où se trouvait cette pièce. " [Entretien avec M. D., 12 juillet 2007].

Des organisations syndicales de personnel aérien se rapprochent alors des associations de défense des droits de l'homme et des droits des étrangers. Un groupe de travail créé en 1987 donne ainsi naissance en 1989 à l'ANAFE (Association nationale d'assistance aux frontières pour les étrangers) ${ }^{3}$ dans l'objectif de coordonner l'action des différentes organisations, et de se poser en interlocuteur du ministère de l'Intérieur. Des articles et des communiqués collectifs dénoncent les pratiques discrétionnaires de la Police de l'air aux frontières et remettent en question la détention administrative comme contraire aux principes de la communauté politique française: «depuis la Déclaration des droits de l'homme et des citoyens de 1789 au moins, nul ne peut être détenu que dans les cas et selon les modalités prévus par la loi » [Julinet, 1999, en ligne]. Cette association s'organise en front de pression pour revendiquer une légalisation des pratiques administratives : elle réclame le contrôle d'un juge lors la procédure de détention à la frontière. L'ANAFE soutient des étrangers détenus, qui, une fois admis sur le territoire, engagent des procédures devant les tribunaux civils. Ces actions conduisent à la condamnation du ministère de l'Intérieur par le tribunal de grande instance de Paris qui, en mars 1992, qualifie la «détention » en aéroport de «voie de fait», c'est-à-dire d'atteinte grave à la liberté individuelle : la décision créé une jurisprudence qui affirme l'illégalité de la détention aux frontières 4 .

En décembre 1991, sous la pression des procédures judiciaires et des mobilisations associatives, le ministre de l'Intérieur socialiste Philipe Marchand introduit, en amendement d'un projet de loi en discussion, un article créant des «zones de transit», qui vise à donner une base légale aux pra-

3 À sa création, l'ANAFE rassemble Amnesty International, l'Association des juristes pour la reconnaissance des droits fondamentaux des immigrés (ADDE), Amnesty International, la Cimade (Service CEcuménique d'Entraide), le COMEDE (Comité Médical pour les Exiles), le CAIF (Conseil des Associations Immigrées en France), France Terre d'Asile, le GAS (Groupe Accueil et Solidarité), le GISTI (Groupe d'Information et de Soutien des Immigrés), la Ligue des Droits de l'Homme, le MRAP (Mouvement contre le Racisme et pour l'Amitié entre les Peuples), le Syndicat CFDT des personnels Air-France, le Syndicat CFDT des personnels des aéroports de Paris, le Syndicat des pilotes de l'aviation civile, le Syndicat unitaire des navigants commerciaux et l'Union régionale Ile-de-France CFDT, et également le Syndicat CFDT de le Police de l'Air aux Frontières.

4 Le 25 mars 1992, le tribunal de grande instance de Paris condamne le ministère de l'Intérieur, jugeant que l'État a "gravement porté atteinte à la liberté » de six demandeurs d'asile en les retenant à l'aéroport de Roissy-Charles-de-Gaulle. L'État est condamné à payer 33000 francs de dommages et intérêts aux intéressés et 1 franc symbolique au Groupe d'information et de soutien des travailleurs immigrés (GISTI). Cette décision affirme l'illégalité de la «zone internationale », où sont placés les étrangers non-admis sur le territoire national. 
tiques de détention frontalière. Le Conseil constitutionnel censure la loi au motif que, par la combinaison de sa durée et du degré de contrainte qu'il revêt, ce régime porte à la liberté individuelle une atteinte qui impose l'intervention d'un juge dans les meilleurs délais pour la contrôler. La loi Quilès du 6 juillet 1992, présentée par le ministre suivant, intègre cette exigence du Conseil constitutionnel (mais elle ne lui sera pas déférée) : elle soumet la prolongation du "maintien » en «zone d'attente» (et non plus « de transit») à l'autorisation d'un juge du tribunal de grande instance. La création des zones d'attente légalise les pratiques de détention frontalière en place depuis la fin des années 1970, en les inscrivant dans une procédure contrôlée par une autorité judiciaire : la détention administrative est vérifiée par un juge après quatre jours, puis tous les huit jours; la durée totale de maintien ne peut pas excéder vingt jours.

La zone d'attente est d'abord un cadre juridique - la condition légale du maintien des étrangers à la frontière - qui annule l'extraterritorialité fictive de la zone internationale. L'institution d'un contrôle juridique normalise la procédure de détention et réintroduit ce qui était dénoncé jusqu'alors comme espace de «non-droit» [Israel, 2003] dans le champ d'action, et de bataille, du droit. L'intervention d'un juge des libertés et de la détention donne ainsi naissance au régime de maintien ; pourtant, il est intégré après-coup dans le dispositif frontalier en place depuis plus d'une décennie. Comment cette pratique juridictionnelle s'est-elle mise en place, comment s'applique-t-elle au quotidien et comment a-t-elle évolué ? Comment se déroulent, concrètement, les audiences ? De quels enjeux le travail des juges est-il investi ? Comment le tribunal s'articule-t-il au dispositif de gestion frontalière mis en place à Roissy? Le chapitre traite ces questions en se fondant sur une enquête de terrain menée entre 2004 et 2007. Le matériau comprend des observations dans la zone d'attente de Roissy (dont l'accès a été négocié dans le cadre d'un travail d'assistance juridique pour l'ANAFE auprès des maintenus), et des observations d'audiences au tribunal de grande instance ainsi qu'une série d'entretiens longs avec trois magistrats exerçant ou ayant exercé la fonction de juge des libertés et de la détention (JLD). À partir de ces observations, le propos est de comprendre comment les pratiques discrétionnaires de l'administration, guidées par des impératifs de contrôle des frontières, sont ressaisies et évoluent dans un cadre légal : quels sont les conditions et les effets de cette normalisation, qui opère une introduction paradoxale de la frontière dans le droit?

\section{Le maintien en zone d'attente et les audiences du « 35 quater »}

Les voyageurs dont l'entrée est refusée et les demandeurs d'asile à la frontière sont d'abord gardés dans des cellules situées dans les postes de 
police de l'aéroport. Cette première détention au sein des terminaux dure normalement quelques heures, au terme desquelles les maintenus qui ne sont pas immédiatement refoulés 5 sont transférés dans un second lieu de détention : le centre «Zapi 3 ». Ce centre fermé, sous contrôle de la police de l'air aux frontières, a été construit en 2001 dans la zone de fret de l'aéroport, au milieu de locaux industriels et d'entreprises de service. Les maintenus conduits en Zapi 3 sont brièvement enregistrés dans les bureaux de police situés au rez-de-chaussée, puis ils sont emmenés au premier étage, réservé à l'hébergement. Il y sont pris en charge par une mission de la Croix-Rouge française qui gère le quotidien du centre, alors que la gestion logistique est sous-traitée à un prestataire de service privé. Zapi 3 est un lieu complexe qui articule deux espaces : le lieu d'hébergement et les locaux administratifs, qui en sont séparés par un double sas. S'y trouvent notamment les bureaux de l'OFPRA (Office française de protection des réfugiés et apatrides) où sont menés les entretiens de demande d'asile, des salles de visite pour les avocats ou les membres de la famille, les bureaux de police, les salles où ont lieu les préparations au refoulement et une pièce qui fait office de cellule d'isolement pour les maintenus ayant résisté (à travers des actions individuelles comme des grèves de la faim ou des mutilations) à leur renvoi ou à leur détention. Le centre, dont l'organisation hybride accumule gestion carcérale et gestion humanitaire, rassemble ainsi dans un même espace les différents acteurs, publics et privés, qui interviennent dans la procédure de maintien.

La première présentation des maintenus devant le juge des libertés et de la détention qui statue au tribunal de grande instance de Bobigny, à quelques dizaines de kilomètres de la Zapi 3, a lieu au quatrième jour de détention. Contrairement à l'examen de l'asile et aux autres étapes du maintien qui sont individualisées, le transfert au tribunal concerne un groupe de maintenus qui sont rassemblés tôt le matin dans une des salles de télévision du rezde-chaussée, puis accompagnés par des équipes de police au tribunal où ils attendent dans le « dépôt » situé au sous-sol le début des audiences. Cellesci sont communément appelée "audiences du 35 quater », du nom de l'article 35 quater de l'ordonnance du 2 novembre 1945, qui a établi le contrôle du juge lors du maintien en zone d'attente (il a depuis été abrogé et remplacé par un article du Ceseda cité plus haut, mais le nom est resté). En fait, ces séances sont mixtes et regroupent les audiences du 35 quater, con-

\footnotetext{
5 La loi instituant les zones d'attente en 1992 prévoyait un délai de 24 heures avant le refoulement des étrangers refusés. Depuis la loi Sarkozy de novembre 2003, ce délai d'un «jour franc » n'est pas systématique, mais il doit être réclamé par les personnes contrôlées, à défaut de quoi elles sont expulsables immédiatement. Le chiffre de ces refoulements immédiats sans examen est difficile à connaître ; le ministère de l'Intérieur les a estimés à un peu plus de 14000 en 2007 (Ministère de l'Immigration, de l'intégration, de l'identité nationale et du développement solidaire, 2008), ce qui correspond à peu près à autant de personnes refoulées immédiatement que de personnes maintenues aux frontières.
} 
in D. Fassin (dir.), Les nouvelles frontières de la société française, Paris, La Découverte, 2010, pp. $437-458$

cernant le maintien en zone d'attente, et celles du «35 bis», concernant la régularité de la procédure de rétention administrative (c'est-à-dire l'interpellation de personnes sans-papiers présentes en France, leur enfermement en local de rétention ou Centre de rétention administrative - CRA et leur expulsion). Les audiences du 35 bis et quater commencent en fin de matinée et se prolongent sans interruption le plus souvent jusqu'en soirée. Elles ont lieu dans une salle de trente à quarante places située directement à l'entrée du tribunal, en face du portique de sécurité. Leur organisation ramène le rituel de la justice à une certaine promiscuité 6 : la salle est petite et confidentielle, en retrait du vaste hall qui donne sur les autres salles d'audience ; les nombreux examens se succèdent à intervalles de dix minutes en moyenne. Les décisions du juge se rendent dans une ambiance relativement bruyante faite de discussions à voix basses, de va-et-vient, de sonneries de téléphone portable. Une dizaine de policiers sont présents dans la salle et peuvent exercer un contrôle sur les allées et venues du public en refusant par exemple l'accès de la salle. Toutefois, la forme du procès, même vidée de certains attributs symboliques, demeure un moment impressionnant aux yeux de beaucoup d'étrangers, qui ne sont pas prévenus de la tenue et des enjeux de l'audience, comme j'ai pu l'observer durant le travail d'assistance juridique. "Je rencontre $M$. N., lors d'une permanence juridique en zone d'attente. Il est 18 h00 lorsqu'il s'assoit, sans un mot, devant mon bureau. Il me tend un papier, qui est son "Ordonnance de prolongation de maintien en zone d'attente", et attend, toujours en silence, des explications. Je lui explique qu'il a été emmené au tribunal aujourd'hui et que le juge a décidé qu'il resterait en zone d'attente. Durant cette explication, plusieurs éléments lui semblent difficiles à comprendre: d'une part, le fait qu'il a eu un avocat (il n'a pas saisi que l'avocat commis d'office plaidant lors de son audience, et qui ne lui a pas adressé la parole, le représentait); d'autre part, le fait que ce n'était pas lui qui était jugé, mais la légalité de sa détention. À la place de sa signature, l'ordonnance comporte la mention 'refuse de signer'. » [Journal de terrain, 5 mai 2005]. Si les maintenus ne possèdent pas déjà une famille ou le contact d'un avocat en France, l'audience pose en effet pour eux un problème d'intelligibilité : l'enjeu du passage devant le juge ne leur est pas précisé, la procédure - au demeurant complexe - est inconnue. L'examen de la procédure administrative peut alors se renverser, en les instituant dans une position peu lisible d'accusé, et en mettant en scène une pénalisation de leur acte.

Toutefois, cette vérification procédurale, parfois très technique, est un

\footnotetext{
6 Ce point a été traité dans une ethnographie consacrée aux audiences [Hamel et Lemoine, 2000]. Ces observations concernent les audiences du 35 bis réservées aux personnes retenues dans les centres de rétention administratives, qui sont, comme je l'ai précisé, regroupées avec celles 35 quater pour les étrangers maintenus en zone d'attente au tribunal de Bobigny.
} 
in D. Fassin (dir.), Les nouvelles frontières de la société française, Paris, La Découverte, 2010, pp. $437-458$

moment essentiel dans le parcours de maintien car le juge peut prolonger la détention administrative mais il peut également admettre les maintenus sur le territoire. En 2006, 25\% des demandeurs d'asiles à la frontière ont été libérés par le juge des libertés et de la détention (JLD) ; 38\% des maintenus admis sur le territoire l'ont été sur décision du JLD, ce qui fait des audiences du 35 quater la cause principale d'admission des étrangers maintenus, devant l'admission au titre de l'asile (qui représente environ 30\% des admissions) ${ }^{7}$. Ainsi, le contrôle de la procédure se présente simultanément sous deux formes : celle d'une garantie légale et formelle qui normalise le dispositif contesté d'enfermement et d'expulsion; mais aussi celle d'une porte de sortie hors de ce dispositif, incluant une tierce logique juridique dans le face-à-face entre l'administration et les étrangers contrôlés. C'est cette ambiguïté que j'aimerais à présent explorer, à travers une observation du déroulement des audiences et de la forme des débats qui les animent. En effet, le jugement participe au travail de sélection et d'admission par lequel le dispositif de maintien remplit sa fonction de frontière. Mais il participe aussi à produire la frontière, comprise cette fois non plus comme point d'accès au territoire mais comme délimitation d'un espace politique national, dans la mesure où le contrôle juridique négocie et redéfinit sans cesse les pratiques de gouvernement acceptables et celle qui ne le sont pas.

\section{Le travail des juges et le travail de l'administration}

«Il est 11h40. Les maintenus devant être présentés à l'audience sont en retard.

Le juge, aux policiers assis dans le fond de la salle : "Vous ne savez pas où ils sont?"

L'avocate de l'administration salue un avocat qui vient d'entrer. Les autres maintenus attendent en silence, les policiers vont et viennent. Trois policiers entrent en accompagnant une femme voilée et trois enfants, dont l'aîné a peut-être 7 ou 8 ans. Les sept policiers parlent entre eux, détendus, puis deux d'entre eux quittent la salle en lançant un "allez bon courage!" à leurs collègues. Un jeune demandeur d'asile qui vient de voir prolonger son maintien en zone d'attente parle avec un des enfants de la maintenue qui est présentée devant le juge. Plusieurs personnes, qui semblent avoir un lien avec cette femme, étaient assises au même rang que moi, près de la porte : elles s'avancent au premier rang à la droite de la maintenue. Un homme âgé entre dans la salle. Un policier se lève et lui demande: "Qu'est-ce que vous voulez? "Il dit un nom. Le policier répond: "Il n'est

\footnotetext{
7 Ces chiffres sont ceux communiqués par le ministère de l'Intérieur aux associations habilitées à intervenir en zone d'attente et rendues public par elles [ANAFÉ, 2008].
} 
in D. Fassin (dir.), Les nouvelles frontières de la société française, Paris, La Découverte, 2010, pp. $437-458$

pas ici », le pousse doucement mais fermement dehors et ferme la porte.

Le juge, au greffier : "Vous lui dites qu'on va commencer. On reprend le même interprète que tout à l'heure? " Le greffier confirme.

Le juge lit le nom de la femme et de ses enfants. Elle est assise avec sa fille sur ses genoux, tandis que ses autres enfants sont debout à ses côtés. L'interprète, à qui la femme s'est mise à parler dès son arrivée, prend la parole : "Dans le village... "

Il est aussitôt coupé par le juge qui continue à résumer : "La maintenue est arrivée le 12 avril et sa demande d'asile a été refusée le 14 avril [...] »

L'avocat de la maintenue prend la parole et énumère plusieurs détails techniques qui, plaide-t-il, annulent la procédure de placement en zone d'attente. Le juge qualifie ces éléments de "petit problème technique ». La parole est donnée à l'avocate de l'administration, qui fait remarquer que la maintenue n'était pas enregistrée comme demandeuse d'asile au moment de son contrôle en aéroport : "Sa demande d'asile ne sera enregistrée qu'une heure plus tard. Je précise au passage qu'actuellement, il y a beaucoup de demandeurs qui obtiennent l'asile. "L'avocate poursuit en rappelant la raison du retard de la maintenue et ses enfants à l'audience. Ceux-ci ne sont pas venus au tribunal avec le reste du groupe des maintenus, mais y ont été escortés depuis l'aéroport immédiatement après une tentative de renvoi qui a échoué : "Elle a fait ce matin l'objet d'un embarquement assez houleux puisqu'elle et ses enfants ont gesticulé en tous sens et que le commandant de bord s'est opposé [à leur renvoi] pour des raisons de sécurité. Je demande que son maintien en zone d'attente soit prolongé pour mener à bien son embarquement, qui s'avère particulièrement difficile. "

L'avocat de la maintenue répond en évoquant le fait que sa cliente, dont la demande d'asile a été refusée (suite à quoi la police a tenté de l'expulser dans la matinée), voudrait donner des "éléments d'information supplémentaires » sur sa demande d'asile.

Il se fait couper rapidement la parole par l'avocate de l'administration : "Les éléments d'information, ben je les connais, je lis les journaux et ceux qui traitent les demandes d'asile politique lisent aussi les journaux! "

L'avocat de la maintenue insiste: "Elle a sa famille réfugiée politique ici. "

Le juge intervient: "Attendez! On n'examine pas sa demande d'asile là... Elle a des garanties de représentation?"

L'avocat lui tend une feuille. Les "garanties de représentation" renvoient à la présence de garants résidant en France, qui s'engagent à prendre en charge la maintenue et fournissent à l'administration une adresse à laquelle elle est joignable. Le JLD peut en effet décider, sans avoir besoin de motiver sa décision, qu'une personne présente des "garanties 》 suffisantes pour que l'administration procède à l'examen de sa situation sans la garder en détention, et que la privation de liberté dans son cas n'est pas nécessaire. 
in D. Fassin (dir.), Les nouvelles frontières de la société française, Paris, La Découverte, 2010, pp. $437-458$

Le juge se met à écrire sa décision, en prenant devant lui le code, qu'il feuilletait avant le début de la séance. Il est 12 h07 ; l'audience a duré 27 minutes.

L'avocate de l'administration prend un dossier et sort de la salle. Le juge mâche du chewing-gum. L'interprète boucle son sac. Les proches venus assister à l'audience parlent entre eux ; certains ont des sacs de cadeaux pour les enfants.

Le juge prononce son ordonnance : "Je prolonge son renouvellement de maintien de 8 jours. Et cette mesure s'applique également à ses enfants." La maintenue sera reconduite par la police en zone d'attente.

L'interprète : "Elle refuse de signer. "

Le juge : "Bon. L'audience est levée. "

Une dame âgée suit la maintenue et ses enfants du regard, les larmes aux yeux. L'interprète continue à traduire, peut-être pour expliquer le maintien en zone d'attente aux proches venus assister à l'audience au premier rang. Quelques femmes et hommes qui semblent connaître la maintenue et sa famille, mais n'ont pas trouvé de place dans la salle, regardent l'audience par la fenêtre. " [Journal de terrain, 16 avril 2007].

Ces observations nous apprennent plusieurs choses. D'abord, comme le rappelle le président de séance à l'avocat de Mme G., le juge n'examine pas « le fond », c'est-à-dire le parcours de la personne, les raisons de sa présence en France ou sa demande d'asile ; il contrôle uniquement la légalité de la procédure de détention administrative. Or malgré le principe de séparation des compétences qui est ici clairement rappelé, les enjeux de la détention frontalière restent présents à l'horizon des audiences, notamment en ce qui concerne le refoulement des demandeurs d'asiles refusés à la frontière. Pendant l'audience de Mme G., l'avocate de l'administration est soucieuse d'exclure le débat de fond portant sur la demande d'asile. Cependant, elle commence par soulever dans son argumentation deux éléments qui ont rapport avec la demande d'asile et qui visent plus précisément à jeter le soupçon sur son bien-fondé. Elle rappelle que Mme G. n'a demandé l'asile qu'une heure après son contrôle. Les maintenus peuvent en effet demander l'asile à tout moment au cours de leur procédure de maintien. Or dans les faits, le ministère de l'Intérieur considère les demandes d'asile qui ne sont pas déposées spontanément ${ }^{8}$ à la frontière comme relevant d'une stratégie dictée par les passeurs, ou par les défenseurs des étrangers, pour éviter un refoulement immédiat (le caractère «tardif » d'une demande d'asile figure ainsi parfois comme un des arguments motivant le refus de la demande). L'avocate de l'administration ajoute par ailleurs qu' " actuellement, il y a beaucoup de demandeurs qui obtiennent l'asile »; or même dans ce con-

8 Dans la pratique, en aérogare, si les mots « je demande l'asile » ou «I want asylum » en anglais ne sont pas prononcés lors de la première interaction, l'étranger contrôlé n'est pas enregistré comme demandeur d'asile. 
in D. Fassin (dir.), Les nouvelles frontières de la société française, Paris, La Découverte, 2010, pp. $437-458$

texte de clémence, la demande d'asile de Mme G. a été jugée « manifestement infondée ». L'évocation de cette demande d'asile, qui a les attributs d'une fausse demande, est suivie par une conclusion qui rappelle les enjeux de la détention : "Je demande que son maintien en zone d'attente soit prolongé pour mener à bien son embarquement qui s'avère particulièrement difficile. ». On voit que si l'examen des demandes d'asile est exclu des débats, le discours administratif sur l'asile, qui est un discours de suspicion, est présent quant à lui et imprègne les débats de façon à la fois continue et implicite. Les arguments avancés par l'avocate sont en effet suggérés et j'ai dû les compléter pour les rendre intelligibles. Cela tient autant au caractère répétitif des débats, que les initiés ont tendance à raccourcir, qu'à la maîtrise, par l'avocate de l'administration, des limites dans lesquelles l'argumentation de fond peut influencer le débat sans faire basculer celui-ci hors du domaine de compétence du juge. La légitimation administrative de la détention et du refoulement se fonde ainsi sur un discours de disqualification et de fraude, qui n'intervient pas de façon explicite dans le travail du juge, mais dessine l'horizon de sa décision. Celle-ci illustre un mécanisme important pour saisir le fonctionnement de la zone d'attente, mais que nous n'aurons pas le loisir d'approfondir ici, à savoir, la façon dont le discours de fermeture des frontières circule dans le dispositif, en se superposant aux différentes logiques, juridiques, humanitaires, commerciales, médicales, qui le composent.

Tandis que les limites de la compétence du juge sont nettement rappelées durant l'audience ("Attendez! On n'examine pas sa demande d'asile là...»), les entretiens avec les magistrats révèlent une pratique qui varie en fonction des acteurs. En effet, la distinction entre la forme de la procédure et le jugement de fond sur le maintien, dont l'examen revient au seul ministère de l'Intérieur, est dans les faits plus ambiguë qu'il n'y parait. Un magistrat rappelle ainsi que les JLD ont accès à la décision motivée de refus de demande d'asile qui figure dans le dossier des maintenus, et il reconnaît que la lecture de ces décisions a pu avoir une influence sur ses jugements. "En l'état du droit, on n'avait pas la possibilité d'apprécier la véracité du récit [d'asile]. Cela dit, des fois, je me suis basé sur la situation individuelle du maintenu. Il m'arrivait d'avoir [à disposition, dans le dossier du maintenu, le document de] refus d'asile du ministère de l'Intérieur basé sur l'avis de l'OFPRA. Des fois, la lecture de la motivation du refus faisait référence à des éléments qui me paraissaient scandaleux: par exemple, sous prétexte que les persécutions n'avaient pas été exercées par le pouvoir en place mais par une faction ${ }^{9}$, on n'autorisait pas les personnes à entrer. Quand ça se

9 L'interprétation de la Convention de Genève par l'OFPRA a en effet tendance à restreinte la définition des persécutions donnant droit à l'asile à celles qui sont exercées par des autorités étatiques ou celles contre lesquelles un État ne peut ou ne veut manifestement 
in D. Fassin (dir.), Les nouvelles frontières de la société française, Paris, La Découverte, 2010, pp. $437-458$

passait dans des situations où l'on sentait que le pays était en guerre, il m'est arrivé de dire: "Puisque je n'ai pas de possibilité d'annuler la procédure, je prends une décision parce que je veux éviter un " traitement inhumain et dégradant » en laissant cette personne repartir à destination d'un pays où elle risque la mort. " Je ne me rappelle plus très bien les termes de mes motivations parce que je sais qu'elles n'ont jamais été acceptées [par la Cour d'appel10]. » [Entretien avec M.B., JLD, 18 août 2007]. Selon cet entretien, le travail de juge des libertés et de la détention, qui se conçoit comme un garant des libertés individuelles, s'appuie sur une évaluation qui dépasse le strict cadre procédural, même si le jugement est ressaisi ensuite à l'intérieur de ce champ d'appréciation et des outils légaux dont disposent les JLD : "Il fallait trouver des subterfuges pour motiver nos décisions, et on se reportait sur des subterfuges juridiques. » [Entretien avec M.B., JLD, 18 août 2007].

Si le JLD peut refuser de prolonger le maintien en zone d'attente, ces décisions sont en général mal appréciées par la police (mais aussi par l'OFPRA), qui estime que la mission de l'administration a été entravée. Comme l'explique une policière à la suite de l'admission au tribunal de grande instance d'un demandeur d'asile malien: "Nous, on fait notre travail, on intercepte les fraudeurs. Et on les emmène au [tribunal] où le juge libère le demandeur (...) on se demande à quoi on sert. L'administration n'a même pas le temps de faire son travail » [Entretien informel, 5 février 2005]. Le travail de l'administration renvoie à un objectif de refoulement des personnes à la frontière. Pour y atteindre au mieux, la police exerce donc un ajustement des délais de détention administrative et d'expulsion afin de renvoyer le plus grand nombre possible de maintenus dans les quatre premiers jours de détention, avant la présentation devant le juge des libertés et de la détention. Un rapport présenté devant le Sénat indique qu'une des évolutions majeures dans la gestion du contrôle frontalier à Roissy concerne l'augmentation sensible des refoulements avant le délai des quatre premiers jours, c'est-à-dire avant la première audience du 35 quater. En effet, en 2003, 43,6\% des personnes placées en zone d'attente étaient toujours maintenues après 96 heures (et donc présentées devant le juge) ; alors qu'en 2005, 14,5\% d'entre elles (soit 2010 étrangers) étaient toujours en zone d'attente après 96 heures [Commission d'enquête du Sénat, 2007]. Si les audiences du 35 quater instituent un encadrement légal de la détention, cet arbitrage se trouve cependant escamoté par le jeu des délais administratifs. Comme le montre le transfert sous escorte de Mme G. et ses enfants après

pas protéger ses ressortissants (ce dernier point, d'ailleurs, est particulièrement difficile à démontrer lors de l'examen).

10 L'administration fait généralement appel de telles décisions, notamment pour ne pas créer de jurisprudence étendant le pouvoir d'appréciation des juges des libertés et de la détention. 
in D. Fassin (dir.), Les nouvelles frontières de la société française, Paris, La Découverte, 2010, pp. $437-458$

une tentative d'expulsion avortée, de l'aéroport au tribunal, puis du tribunal en Zapi 3, les audiences n'occupent pas vraiment la position de surplomb que le texte de loi leur prêtait à la création des zones d'attente, mais apparaissent plutôt comme une pièce instable dans le dispositif frontalier orienté vers l'objectif d'expulsion.

\section{Prendre le contentieux des étrangers au sérieux : l'institution des audiences}

Si le contrôle du juge sur la procédure de maintien en zone d'attente est prévu dès 1992, son application ne ressemble pas, dans un premier temps, à l'audience décrite plus haut. Les audiences du 35 quater, dans leur forme actuelle, sont en effet le fruit de deux évolutions majeures dans la pratique juridictionnelle : l'institution de séances publiques dans des salles du tribunal d'une part, et la création de la fonction spécialisée de juge des libertés et de la détention d'autre part. Jusqu'à la fin des années 1990 en effet, les juges statuaient sur le droit des étrangers dans leurs bureaux. Un magistrat en fonction à cette époque se souvient de ces audiences comme d'un travail expéditif et administratif : le public en est absent, les avocats connaissant le contentieux des étrangers, rares, et le nombre de dossiers accumulés très important, ce qui aboutit à des délais d'audience de quelques minutes (3 à 5) en moyenne. "Quand on statuait sur le droit des étrangers à l'origine, on statuait dans notre bureau, [...] on interdisait aux gens d'y accéder. [...] Il y avait un procédé, c'est que les portes des bureaux étaient ouvertes...à un étage où personne n'allait! Et les policiers (à P. en tout cas) empêchaient les gens d'accéder aux bureaux dans lesquels on rendait des décisions. [...] Il y avait 60 dossiers par jour. " [Entretien avec Mme E., JLD, 30 mars 2005]. Si la reformulation législative du maintien en zone d'attente introduit l'intervention d'un juge, la mise en pratique de ce principe est marquée par un effort de dénégation, à travers des conditions d'application du droit (le choix des lieux, le déroulement des audiences) qui sont anormales par rapport aux coutumes de la justice. Ces conditions invitent à réfléchir sur la place et la fonction des audiences dans le dispositif frontalier. Analysant les modalités d'appréciation des juges dans le contentieux des étrangers, le magistrat Denis Salas estime ainsi que « le juge est une tête d'épingle dans un dispositif dont il assure le bouclage [...] À sa marginalité opérationnelle correspond le seul effet attendu de sa présence: la légitimation du système » [Salas, 2003, p. 80-81]. Toutefois, les conditions et les enjeux de cette juridiction se sont complexifiés depuis une décennie.

La première évolution significative est l'institution d'audiences publiques. Cette réorganisation a été décidée par l'administration judiciaire à la demande des juges, interpellés par des associations de défense du droit 
des étrangers, comme le GISTI. La mobilisation associative pour la mise en place d'audiences publiques révèle une évolution dans les pratiques d'opposition à la détention des étrangers et les usages du droit. Il ne s'agit plus de contester la légalité des pratiques policières aux frontières, comme ce fut le cas avant 1992, mais de garantir les conditions d'exercice d'un contrôle juridique sur ces pratiques. Dans ce cas précis - qui vient s'ajouter aux autres formes de contestation sans les remplacer - les résistances à la détention frontalière se déplacent sur le terrain de la justice. Il s'agit de faire avancer la question spécifique du maintien en zone d'attente en s'appuyant sur des évolutions de la pratique du droit ${ }^{11}$, c'est-à-dire de désenclaver le contentieux des étranger de son isolement et de son opacité, et de la réhabiliter au sein de l'organisation judiciaire. En réclamant la publicité des audiences, la mobilisation associative, qui se fonde sur une demande d'encadrement juridique depuis ses origines en 1989, cherche ainsi à modifier les conditions d'application de cet encadrement pour redéfinir l'intervention des juges dans le dispositif de maintien.

Le juge B., membre du Syndicat de la Magistrature (syndicat impliqué dans les mobilisations contre la détention administrative des étrangers depuis les années 1970), revient ainsi sur la mise en place d'audiences publiques du 35 quater au tribunal de grande instance de Bobigny : " $C$ 'est l'évolution du droit, ce sont les pressions qui on fait que ces audiences ont été publiques, il a même fallu attendre une décision de la Cour d'appel de Paris qui a considéré que les audiences devaient êtres publiques, parce qu'à l'époque elles ne l'étaient pas. S'il n'y avait pas eu ces interventions persistantes de gens qui disaient: "On doit pouvoir vous voir en train de juger », on en serait encore à statuer dans des bureaux [...] Quand moi je suis arrivé à $B$., il y avait des salles d'audience, on était en robe, on prenait ce contentieux au sérieux, ce n'était pas en catimini derrière une porte d'un bureau. » [Entretien avec M. B., JLD, 18 août 2007]

\section{Investir la jurisprudence : les juges des libertés et de la détention}

Si l'institution d'audiences publiques est le fruit d'une bataille juridique, la seconde évolution qui marque les audiences du 35 quater, quant à elle, ne vient pas d'une mobilisation sur le terrain du droit des étrangers, mais de la répercussion sur ce terrain d'une réorganisation générale qui touche le monde judiciaire. Avec la création de la fonction de juge des libertés et de la

\footnotetext{
11 Les travaux sur la «cause lawyering » (la défense juridique de causes politiques, sociales, environnementales, économiques) proposent une réflexion en ce sens sur ce que les mobilisations juridiques font au droit [Sarat et Scheingold, 1998, p. 3-31].
} 
in D. Fassin (dir.), Les nouvelles frontières de la société française, Paris, La Découverte, 2010, pp. 437-458

détention (JLD) par la loi Guigou du 15 juin 2000, les décisions concernant des situations de privation de liberté, comme la détention provisoire, la rétention ou le maintien en zone d'attente, deviennent toutes de la compétence des juges des libertés et de la détention. Cette fonction, conçue spécifiquement pour garantir le respect des libertés fondamentales, est confiée à des magistrats d'un niveau de hiérarchie et d'expérience élevés. Cette spécialisation du contrôle juridique introduit des changements dans l'exercice du droit des étrangers. Les audiences du 35 quater sont menées par une équipe restreinte de magistrats expérimentés, qui sont complètement dédiés à cette fonction. Ceux-ci ont ainsi la possibilité d'amorcer une réflexion collective sur la jurisprudence ou de travailler à une harmonisation de leurs décisions, dans le cadre étroit de leur compétence. Or, si les JLD maîtrisent et «investissent désormais la jurisprudence » sur la détention frontalière comme le relève le juge B., leur intervention reste soumise à une pression administrative. La marge d'appréciation des JLD est ainsi surveillée à travers des rappels à l'ordre plus précis et plus personnels, en faisant circuler par exemple des listes nominales de juges présentés comme trop favorables aux étrangers dans leurs jugements : " [...] il y a des pressions directes sur les magistrats puisque je me rappelle très bien qu'il y avait des interventions de la part de la préfecture auprès du président du tribunal pour protester contre une décision qui avait été prise par des juges. [...] Il y a 2 ans, [les juges de la Cour d'appel de Paris] avaient fait une note dans laquelle ils avaient compilé toutes les décisions qu'ils avaient infirmées des magistrats du tribunal de grande instance et ils avaient demandé au président du tribunal de diffuser cette note pour stigmatiser les juges. " [Entretien avec M.B., JLD, 18 août 2007]

Les nouvelles conditions de la prise de décision, déléguée à des juges maîtrisant le contentieux, assurés par un niveau de hiérarchie et une expérience solides, génèrent également de nouvelles réponses administratives, qui s'adaptent au caractère spécifique (personnel et hiérarchique) de la décision. Ce faisant, l'administration, ici la préfecture, qui gère la détention frontalière, intervient sur le terrain juridique, en rappelant, contre le travail jurisprudentiel des JLD, les interprétations du droit qui doivent faire règle, c'est-à-dire ceux de la Cour d'appel de Paris, historiquement défavorable au droit des étrangers. Ainsi le contrôle juridique est-il plus qu'une «tête d'épingle » qui vient verrouiller et légitimer le dispositif de détention aux frontières ; mais son exercice reste pris dans des jeux d'autorité et une promiscuité quotidienne qui rendent l'articulation entre les audiences et le dispositif de maintien complexe, oscillant entre un regard exercé sur les pratiques policières et leur normalisation à travers un exercice du droit pénétré par les logiques administratives.

Délocaliser la justice : le 35 quater en Zapi 3 
Dans ce contexte, l'organisation des audiences est marquée par une certaine plasticité et incertitude : elle a connu des changements importants et continue à faire l'objet de projets de réorganisation. Une évolution législative prévoit depuis 2003 l'aménagement de salles d'audience hors du tribunal de grande instance, à l'intérieur de la zone d'attente. Toutefois, cette délocalisation, prévue en fait dans les plans de construction du centre depuis 2001, n'a pas encore été mise en place. La salle d'audience en Zapi 3 comprend un système de vidéoconférence pour diffuser les examens au tribunal de grande instance et souscrire au principe de publicité des audiences. Elle a été construite après l'inauguration du centre d'hébergement dans la partie administrative de la Zapi 3. Depuis, elle reste fermée : l'idée de localiser l'activité de la justice dans la zone d'attente a fait l'objet d'une vive polémique dans les milieux professionnels du droit 12 et le décret en établissant l'usage n'est pas encore paru. L'inclusion du contentieux des étrangers à l'intérieur du lieu de détention a déjà été mise en place dans d'autres cas, comme au Centre de rétention administrative (CRA) de Coquelles depuis juin 2005. Elle se justifie par deux discours : l'un, administratif, de rationalisation des coûts de gestion, car la délocalisation des audiences supprime notamment les nombreuses escortes policières; l'autre, humanitaire, d'amélioration des conditions de détention par la suppression des allers et retours sous escorte vers le tribunal et de l'attente dans le dépôt (la CroixRouge a ainsi pris position pour la délocalisation du tribunal qu'elle juge «plus humaine ${ }^{13}$ ). Mais au-delà des considérations logistiques, le projet de déplacer les audiences en Zapi 3 rappelle comment, depuis la création des zones d'attente, un enjeu de clôture du dispositif se joue autour du lieu et de la forme assignés au jugement des étrangers.

Lorsque cette question a été abordée lors de mes entretiens de terrain, souvent à l'initiative des magistrats qui restent vivement attachés à la conservation des salles d'audience à Bobigny, deux niveaux de réponse pouvaient être identifiés. Au niveau des principes, les magistrats rappelaient l'importance de la publicité des audiences en tribunal, garante de la souveraineté de la justice, rendue par les juges « au nom du peuple français ». Au niveau du travail quotidien, les magistrats redoutaient les effets de détermination du jugement recherchés par le déplacement des audiences à la frontière, en insistant sur l'influence du lieu (la disposition sécuritaire, l'encadrement policier) sur le jugement rendu. Le juge B., qui s'est opposé en 2003 à la loi de délocalisation des audiences à travers son action syndicale, explique les raisons de cette mobilisation, partagée par les différents

12 Voir Van Eeckhout, L. (2005), « Polémique autour de la délocalisation des tribunaux pour étrangers », Le Monde, 1 er Juillet 2005.

13 Voir « Une salle d'audience contestée », Le Parisien, 14 décembre 2003. 
in D. Fassin (dir.), Les nouvelles frontières de la société française, Paris, La Découverte, 2010, pp. $437-458$

syndicats de magistrats au-delà de leur orientation politique [Rosenczveig, 2002] et relayée par le président du tribunal : "[Les salles d'audience en Zapi 3] étai[en] tévidemment des lieux qui étaient totalement inaccessibles. Ne pouvaient y être que des gens qui travaillaient sur place. Donc le premier inconvénient, celui qui me paraissait le plus important tout de même, c'est que la justice ne peut s'exercer qu'à partir du moment où elle s'offre au regard du citoyen. Si on veut un minimum de contrôle sur l'activité du juge, il faut que le public puisse y avoir accès. [...] La visibilité comme accessibilité à la façon dont opère la justice, pour moi, c'est essentiel dans le travail du juge. C'est quelque chose avec lequel il faut composer. Si on travaille dans notre coin, je peux vous garantir que quelles que soient les précautions prises, évidemment il y aura des abus. Par ailleurs, le statut même de ces centres dans lesquels on va tenir des audiences n'est pas très clair, parce que ce ne sont pas des locaux qui appartiennent au ministère de la Justice. Ce sont des locaux qui sont la propriété du ministère de l'Intérieur : ce ne sont pas des lieux neutres... Que les juges statuent dans des lieux qui ne sont pas des lieux de justice, ça me pose énormément de problèmes; de la même façon que statuer dans des gymnases, comme on a pu le faire à un moment dans le procès d'islamistes - parce que soi-disant il y a avait trop de monde - ce n'était pas normal. Il faut que la justice soit rendue dans des lieux de justice, identifiés comme tels et identifiables. Peu importe la façon dont les juges ressentent les choses. Avec un peu de chance, peut-être qu'effectivement les décisions seront correctes; mais on ne peut pas compter sur la seule vigilance du magistrat. Ce qui est très important, c'est l'apparence dans laquelle s'exerce la justice. " [Entretien avec M.B., JLD, 18 août 2007].

\section{Conclusion}

La défense de la publicité des audiences, commune aux mobilisations associatives et aux mobilisations du monde judiciaire, est ce qui donne naissance aux audiences du 35 quater ; mais c'est également ce qui problématise leur évolution à venir, et leur possible délocalisation à l'intérieur des zones d'attente. Pour les magistrats interrogés, la définition de la justice qui soustend ce principe de publicité propose une réévaluation des rapports entre État, citoyenneté et nationalité : du moment qu'il y a un rapport de gouvernement entre l'État et des individus, ces derniers ne peuvent pas être considérés hors des cadres de la citoyenneté. « À partir du moment où vous jugez dans une zone de fret, ça veut très clairement dire que vous considérez qu'il y a une catégorie de citoyens qui n'ont pas le droit à la publicité [...] l'horizon idéal pour nous c'est de faire en sorte que les étrangers ne soient pas considérés comme des justiciables à part, qu'ils bénéficient de procédures 
qui soient des procédures équitables qui leur permettent de faire valoir leurs droits, et pas ce type de procédures où avant même de pouvoir entrer sur le territoire français, leur sort est scellé. " [Entretien avec M.B., JLD, 18 août 2007].

Le fait de désigner spontanément les étrangers contrôlés à la frontière comme « une catégorie de citoyens » et l'équivalence entre "citoyen » et « justiciable» impliquent une idée de la citoyenneté qui ne se rabat pas sur les frontières tracées de la nationalité. Étienne Balibar invitait à une telle réévaluation en proposant une définition non territoriale de la citoyenneté comprise comme « communauté de destin » [Balibar, 1992, p. 61]. Le juge B. emploie, quant à lui, le mot de « citoyen » pour désigner celui qui entre dans sa juridiction. Cet usage de la citoyenneté prend position sur la question des frontières de l'État de droit en affirmant qu'il n'y a pas d'extériorité possible du pouvoir étatique au droit, ni de lieu où le pouvoir étatique déborde le droit - fut-ce la frontière ${ }^{14}$. C'est l'inclusion dans la pratique juridique qui confère au sujet la qualité de citoyen. Dans cette vision, la pratique juridique et les droits fondamentaux sont indissociables et s'impliquent nécessairement l'un l'autre. Dans l'approche normative de la nation, comme « territoire du droit» [Fischer, 2006], les frontières deviennent un processus politique centrifuge (d'inclusion), un lieu où se négocie la coïncidence entre les droits de l'homme et ceux du citoyen; mais c'est aussi un lieu où les seconds sont éventuellement posés comme garantie et condition d'application des premiers.

\section{Bibliographie}

\section{Rapports et documents}

ANAFE (2008), Statistiques relatives aux étrangers à la frontière. Novembre 2008, http://www.anafe.org/download/generalites/stats-za-nov2008.pdf (consulté le 2/08/2009).

Gisti (1991) «Roissy : un filtrage sélectif », Plein Droit, $\mathrm{n}^{\circ}$ 13, http://www.gisti.org/doc/plein-droit/13/roissy.html (consulté le 17/08/2009).

14 Le fait que la frontière soit soumise aux pratiques gouvernementales régulières d'un pays n'a rien d'évident. Dans son ouvrage sur le passeport et les droits du passage, Marc Salter s'appuie sur les pratiques de passage des frontières dans les cités médiévales pour examiner comment la frontière a longtemps été un espace où le sujet était soumis au pouvoir de l'État dans lequel il cherchait à entrer [Salter, 2003, p. 127]. 
in D. Fassin (dir.), Les nouvelles frontières de la société française, Paris, La Découverte, 2010, pp. $437-458$

JouRnal OFFICIEL (2009), Code de l'entrée et du séjour des étrangers et du droit d'asile (Ceseda), Les Journaux officiels, Paris.

Loi n 92-625 du 6 juillet 1992, Journal officiel, 9 Juillet 1992.

Loi n ${ }^{\circ} 1119$ du 26 novembre 2003, Journal officiel, 27 novembre 2003.

MinisTERE DE L'IMMIGRATION, DE L'INTEGRATION, DE L'IDENTITE NATIONALE ET DU DEVELOPPEMENT SOLIDAIRE, (2008) Réunion annuelle sur le fonctionnement des zones d'attente. Article R. 223-14 du CESEDA. 3 juin 2008, http://www.anafe.org/download/generalites/CR-reunion-annuelleZA-2007.pdf (consulté le 2/08/2009).

Ordonnance $n^{\circ}$ 45-2658 sur les conditions d'entrée et de séjour des étrangers en France du 2 novembre 1945, Journal officiel, 4 Novembre 1945.

OTHILY, G., \& BufFET, F.-N., (2006) Immigration clandestine : une réalité inacceptable, une réponse ferme, juste et humaine, Rapport de la commission d'enquête du Sénat sur l'immigration clandestine, Journal officiel, 7 avril 2006.

\section{Références}

ANDRIJASEVIC, R., (2006) « How to Balance Rights and Responsibilities on Asylum at the EU's Southern Border of Italy and Libya », Centre on Migration, Policy \& Society, University of Oxford, http://www.compas.ox.ac.uk/publications/Working\%2520papers/wp-0627.shtml (consulté le 09/04/2009).

ARENDt, H., (2002 [1951]) Les Origines du totalitarisme, Gallimard, Paris. BAlibar, E., (1992) Les Frontières de la démocratie, La Découverte, Paris.

BAUER, P., \& DARley, M., (dir.) (2007) Frontières de l'Union Européenne : franchissements et résistances, Cefres, Prague.

Courau, H., (2007) Ethnologie de la Forme-camp de Sangatte: de l'exception à la régulation, Archives contemporaines, Paris.

FASSIN, D., (2001) «The biopolitics of otherness: Undocumented foreigners and racial discrimination in French public debate », Anthropology Today, vol. 17 , p. 3-7.

FISCHER, N., (2005) «Clandestins au secret. Contrôle et circulation de l'information dans les centres de rétention administrative français », Cultures et Conflits, $\mathrm{n}^{\circ}$ 57, p. 91-118.

FischER, N., (2006) «Les territoires du droit », Vacarme, n³4, http://www. vacarme.eu.org/article538.html (consulté le 10/02/2009).

ISRAEL, L., (2003) « Faire émerger le droit des étrangers en le contestant, ou l'histoire paradoxale des premières années du GISTI », Politix, $\mathrm{n}^{\circ} 16, \mathrm{p}$. 115-144.

JULINET, S., (1999) « Dans les zones d'attente : atteinte aux libertés et inefficacité », Plein Droit, $\mathrm{n}^{\circ} 44$, http://www.gisti.org/doc/pleindroit/44/atteintes.html (consulté le 17/02/2009). 
in D. Fassin (dir.), Les nouvelles frontières de la société française, Paris, La Découverte, 2010, pp. $437-458$

Kleinman, A., Das, V., Ramphele, M. \& Reynolds, P., (Dir.) (2000) Violence and Subjectivity, University of California Press, Berkeley.

Kobelinsky, C., \& MAKaremi, C., (dir.) (2009) Enfermés dehors. Enquête sur le confinement des étrangers, Editions du Croquant, Bellecombes-enBeauge.

MAKAREMI, C., (2008) «Étudier et assister les étrangers aux frontières », in Fassin, D., \& Bensa, A., (dir.) Les Politiques De L'enquête : Epreuves Ethnographiques, La Découverte, Paris.

MAKAREMI, C., (2008) «Les 'zones de non-droit' : un dispositif pathétique de la démocratie », Anthropologie et Société, $\mathrm{n}^{\circ} 32, \mathrm{p} .81-98$.

MARES, P., (2001) Borderline: Australia's Treatment of Refugees and Asylum Seekers, Sydney, UNSW Press.

PanZani, A., (1975) Une Prison clandestine de la police française, Arenc, François Maspero, Paris.

PratT, A., (2005) Securing Borders: Detention and Deportation in Canada, UBC Press, Vancouver.

Rahola, F., (2007) «La forme-camp. Pour une généalogie des lieux de transit et d'internement du présent », Cultures \& Conflits, $\mathrm{n}^{\circ}$ 68, p. 31-50.

RANCIERE, J., (1998) Aux bords du politique, Gallimard, Paris.

RosenczVeIG, J.-P., (2002) « Droit des étrangers : la délocalisation du TGI de Bobigny », Recueil Dalloz, n 8, 20 février 2002.

SALAS, D., (2003) « Immigration illégale et pratiques judiciaires en France: incriminés, discriminés $"$, Hommes \& Migrations, $\mathrm{n}^{\circ} 1241$, p.78-88.

SAlter, M. B., (2003) Rights of Passage: The Passport in International Relations, Lynne Rienner Publishers, Boulder.

Sarat, A., \& Scheingold, S. A., (1998) Cause Lawyering: Political Commitments and Professional Responsibilities, Oxford University Press, New-York.

VAn gunsteren, H., (1998) A Theory of Citizenship: Organizing Plurality in Contemporary Democracies, Westview Press, Boulder.

Welch, M. \& Schuster, L., (2005) « Detention of Asylum Seekers in the US, UK, France, Germany, and Italy: A Critical View of the Globalizing Culture of Control », Criminology and Criminal Justice, $\mathrm{n}^{\circ}$ 5, p. 331355.

Willen, S. S., (2008) «L'hyperpolitique du 'Plus jamais ça' : Demandeurs d'asile soudanais, turbulence gouvernementale et politiques de contrôle des réfugiés en Israël », Cultures \& Conflits, $\mathrm{n}^{\circ}$ 71, p. 93-113. 
in D. Fassin (dir.), Les nouvelles frontières de la société française, Paris, La Découverte, 2010, pp. 437-458 